\title{
The Role of Poverty in Antimicrobial Resistance
}

\author{
Margaret B. Planta, MD
}

Antimicrobial resistance is a worldwide problem that has deleterious long-term effects as the development of drug resistance outpaces the development of new drugs. Poverty has been cited by the World Health Organization as a major force driving the development of antimicrobial resistance. In developing countries, factors such as inadequate access to effective drugs, unregulated dispensing and manufacture of antimicrobials, and truncated antimicrobial therapy because of cost are contributing to the development of multidrug-resistant organisms. Within the United States, poverty-driven practices such as medication-sharing, use of "leftover" antibiotics, and the purchase and use of foreign-made drugs of questionable quality are likely contributing to antimicrobial resistance. However, there is currently a dearth of studies in the United States analyzing the socioeconomic and behavioral factors behind antimicrobial resistance in United States communities. Further studies of these factors, with an emphasis on povertydriven practices, need to be undertaken in order to fully understand the problem of antimicrobial resistance in the United States and to develop effective intervention to combat this problem. (J Am Board Fam Med 2007;20:533-539.)

Because the development of drug resistance outpaces the development of new drugs, antimicrobial resistance has become a worldwide problem that has deleterious long-term effects. Much study and intervention has focused on the surveillance of hospitals and on the education of physicians, but little research has been done in the United States regarding the role of poverty in antimicrobial resistance.

According to the World Health Organization, "more than any other issue, poverty and inadequate access to drugs continue to be a major force in the development of resistance." ${ }^{1}$ In developing countries, the role of poverty in antimicrobial resistance has been recognized and studied. ${ }^{1-3}$ Multiple poverty-driven factors that contribute to the development of multidrug-resistant organisms have been identified, some of which may be directly affecting resistance in the United States.

Poverty probably plays a role in antimicrobial resistance within the United States as well, but little research has occurred in this area. Socioeconomic

This article was externally peer reviewed.

Submitted 18 January 2007; revised 6 March 2007; accepted 14 March 2007.

From the Sutter Medical Group, Roseville, CA.

Funding: none.

Conflict of interest: none declared.

Corresponding author: Margaret B. Planta, MD, 3100 Douglas Blvd., Roseville, CA 95661 (E-mail: mplanta@pol.net). and behavioral studies of antimicrobial practice, knowledge, attitudes, and beliefs within various poverty-stricken communities in the United States may yield insight into a contributing yet understudied factor in antimicrobial resistance and may lead to more effective interventions to combat resistance.

\section{Poverty and Resistance in Developing Countries}

In developing countries with high mortality, infectious diseases remain the main cause of death. ${ }^{4}$ In 1990 , an estimated $78 \%$ of the world's population lived in developing countries. In those countries, $23 \%$ of deaths were attributable to infectious and parasitic disease. ${ }^{5}$

Resistant bacteria have emerged in these developing countries. In 1996, in Bangladesh, over 95\% of Shigella dysenteriae isolates were resistant to ampicillin, co-trimoxazole, and nalidixic acid, and up to $40 \%$ were resistant to mecillinam. ${ }^{6}$ In Quetta, Pakistan, 69\% of Salmonella typhi isolated from blood were multidrug resistant. ${ }^{7}$ In tropical countries, there has been an emergence of Streptococcus pneumoniae that is resistant to penicillin, cefotaxime, and chloramphenicol. ${ }^{8}$ Neisseria gonorrboeae has developed strains resistant to penicillin, sulfonamides, tetracyclines, and fluoroquinolones. ${ }^{9}$

This problem of multidrug-resistant organisms in developing countries can also directly affect and 
threaten more developed countries (such as the United States) because international travel, driven by globalized trade, allows for easier dissemination of these strains. For example, penicillin-resistant and multidrug-resistant pneumococci, like the serotype $23 \mathrm{~F}$ clone, have been found not only in Mexico, South Africa, South Korea, and Croatia, but also in Portugal, France, and the United States. ${ }^{10}$

Reasons for multidrug-resistant organisms in developing countries are numerous, but the inadequate access to effective drugs, the unregulated manufacture and dispensation of antimicrobials, and the lack of money available to pay for appropriate, high-quality medications are some of the major poverty-driven factors contributing to antimicrobial resistance..$^{1-3}$

In some developing countries, regulation of the manufacture of antibiotics may not exist to any extent that would assure the quality and potency of the medications. A 500-mg capsule of ciprofloxacin that was acquired locally in Vietnam was analyzed and found to contain the equivalent of only $20 \mathrm{mg}$ of ciprofloxacin. ${ }^{2}$ Studies conducted in several other developing countries have also demonstrated counterfeit drugs with few or no active ingredients. ${ }^{3}$

Many developing countries allow the dispensation of antibiotics without a prescription; this can lead to self-medication and dispensation of drugs by untrained people. In one survey from the Rajbari district of Bangladesh, 100,000 doses of antibiotics had been dispensed without a prescription in 1 month. ${ }^{11}$ In another study from Bangladesh, $92 \%$ of medications dispensed by pharmacies were dispensed without a prescription. ${ }^{12}$ In Manila, Philippines, a survey of drugstores showed that $66 \%$ of antibiotic purchases were made without a prescription. ${ }^{13}$ The ease of obtaining an antibiotic without a prescription was directly experienced by this author, who was able to purchase antimicrobials without a prescription from local pharmacies in both Lahore, Pakistan and Iquitos, Peru.

Poverty-stricken patients may forgo the cost of a physician consultation and self-medicate. ${ }^{3}$ They may be more likely to purchase the least expensive (and possibly least potent drug) under the assumption that they were all bioequivalent. Furthermore, these people may only complete a truncated course of therapy because of their inability to pay for the full course of medications. ${ }^{1,3,13}$ Such inappropriate use of antibiotics for inadequate periods of time can exert strong selective pressures on bacterial populations and can contribute to resistance.

\section{Poverty-Driven Practices in the Unites States}

Although it is one of the wealthiest nations in the world, the United States is home to 37 million people living below the poverty line; 46.6 million are without health insurance. ${ }^{14}$ There are also 35.9 million seniors (aged 65 and over), 10\% of whom live in poverty, with many others having limited income and prescription coverage. ${ }^{15}$ For these people, the cost of pharmaceuticals can be prohibitive.

Through limited research and anecdotal experience, it is known that some seniors and low-income patients obtain antimicrobials from other countries and may engage in the sharing of medications. ${ }^{16-18}$ Some save antibiotics from regimens that they did not complete (so-called "leftover" antibiotics) and will take them at the onset of upper respiratory infection symptoms. ${ }^{19}$

Such self-medication can lead to antimicrobial resistance because of the inappropriate use of antibiotics for viral illness, the inappropriate choice of medication for the specific organism, and the inappropriate dose or duration of therapy. Unfortunately, such practices are probably more widespread than realized. McKee et $\mathrm{al}^{18}$ surveyed an ethnically diverse population in an urban setting regarding antibiotic use in the setting of an upper respiratory infection. They found that $26 \% \mathrm{ob}-$ tained antibiotics from a source other than a physician's prescription. Larson et $\mathrm{al}^{17}$ reported that, in a largely Hispanic neighborhood in Manhattan, antibiotics were readily available over the counter at local bodegas (small stores). They also reported the sharing of antibiotics among family members or between friends. In one survey of emergency room patients, $17 \%$ reported taking "leftover" antibiotics for upper respiratory infection symptoms. ${ }^{19}$

As health care and prescription costs soar, more and more people with limited incomes may turn to foreign countries for more affordable medications. A survey of adults in Los Angeles County showed that $14 \%$ had crossed the border into Mexico for health care. Nearly $80 \%$ of those were uninsured. Of the $28 \%$ who purchased medications in Mexico, $90 \%$ were uninsured. Many of these purchases were of antibiotics that were readily available without a prescription. ${ }^{16}$ 
Some people with low income may purchase discounted drugs via the internet. This contributes to antimicrobial resistance through the use of suboptimal-quality drugs. Between 1992 and 1994, $51 \%$ of counterfeit drug cases uncovered by the World Health Organization carried no active ingredient, $17 \%$ contained the wrong ingredient, and $11 \%$ contained less than the recommended concentration of active ingredient. Furthermore, 70\% of these counterfeiting cases were discovered in developing countries. ${ }^{1}$ In the summer of 2003, the Food and Drug Administration examined 1153 shipments that arrived at Miami, New York, and 2 California mail facilities. Of these, $88 \%$ contained unapproved drugs from various countries; $16 \%$ were from Canada, 14\% were from India, 14\% came from Thailand, and $8 \%$ came from the Philippines. $^{20}$

Surveillance studies throughout the United States reveal the emergence of multidrug resistance in common infectious organisms. One study showed Streptococcus pneumoniae resistance to penicillin, ceftriaxone, erythromycin, clindamycin, tetracycline, and trimethoprim-sulfamethoxazole. Approximately $22 \%$ of those isolates were multidrug resistant. There was also a trend toward increased fluoroquinolone resistance. ${ }^{21}$ Neisseria gonorrboeae has shown increasing resistance to penicillin and tetracycline and an emerging resistance to fluoroquinolones. ${ }^{22}$ In 2002, Salmonella that was resistant to amoxicillin/clavulanate, ampicillin, cefoxitin, ceftiofur, cephalothin, chloramphenicol, streptomycin, sulfamethoxazole, and tetracycline was detected in 5 states. $^{23}$

Appropriately, much of the blame for the emergence of resistance in the United States has been placed on inappropriate antibiotic prescribing practices by clinicians and on poor patient compliance. However, the poverty-driven practices of medication sharing, self-treatment, and use of poor-quality, foreign-made drugs are also likely contributors to multidrug-resistant organisms. Unfortunately, there is currently a dearth of research into how these practices directly affect resistance patterns within a community in the United States.

A causal relationship is suggested by the results of research conducted on the resistance of Escherichia coli in a community along the border of the United States and Mexico, where crossing the border to obtain medications is not unusual. In this community, resistance rates were higher than the national average. ${ }^{24} \mathrm{~A}$ better understanding of the impact that these practices have on resistance can lead to an understanding of whether interventional efforts to reduce these practices can lead to a significant reduction of antimicrobial resistance in the community.

\section{Discussion}

Analysis of the information above indicates that poverty may play a role in antimicrobial resistance worldwide. It further suggests that effective intervention cannot be done in isolation. Because of international travel, multidrug-resistant organisms in one country can be more easily disseminated to another country. The ease of obtaining antibiotics without a prescription in some developing countries can lead to self-medication, not only in those developing countries, but in the United States as well. In addition, because of the internet, lowquality and counterfeit drugs affect not only the country of origin, but also populations in the United States. The United States cannot effectively combat antimicrobial resistance within its borders if it ignores the contributing factors from developing countries, and it cannot design effective intervention if it fails to study and recognize the poverty-driven socioeconomic and behavioral factors that may contribute to resistance.

\section{Recommendations for Further Action}

One can argue that antimicrobial resistance as a global health issue is as important as or even more important than global warming because the effects are more immediate (ie, death from infection) and are occurring within this lifetime. Governments of industrialized nations have recognized global warming as a serious health issue and have held summits to formulate ways to combat the problem, yet there has been no international governmental summit to address antimicrobial resistance.

Because of the cost and enforcement issues involved in combating the socioeconomic forces that contribute to antimicrobial resistance, the medical community alone cannot take on this problem and must harness the resources of governments. The power of the medical community in changing public policy and opinion is evidenced by its role in curbing tobacco use. It was not until organized medicine made a formal statement about the negative health effects of tobacco did public policy and 
opinion turn away from the tobacco industry and lead to major financial payments and restrictions in advertising in the United States. It is the role of the medical community in every country to educate its government about the danger of antimicrobial resistance and to advocate for cooperation between governments and for the allocation of resources necessary to implement effective intervention.

Successful multinational cooperation is possible, as evidenced by the containment of severe acute respiratory syndrome (SARS), and by the current efforts to forestall a bird flu pandemic. Worldwide cooperative efforts have eliminated smallpox, and the elimination of polio is within reach. Combating and preventing multidrug-resistant organisms is realizable, provided that nations are committed to making an aggressive effort to address this problem. And because poverty-driven issues in developing countries may directly affect resistance in the United States, the United States needs to take a lead role in addressing this problem from a global perspective.

Based on the review of the literature and current knowledge regarding poverty-driven practices that may contribute to resistance, the following recommendations for global intervention are made:

\section{Restrict over-the-counter dispensing of antimicrobials without a prescription in all countries}

Although evidence is limited, there are some studies that suggest that reducing antibiotic usage may reduce resistance. Investigators in Finland have shown a reduction in prevalence of erythromycin resistant Streptococcus pyogenes after a national campaign to reduce macrolide usage. ${ }^{25}$ Investigators in Iceland showed the disappearance of a multidrugresistant strain of Pneumoccoci after 10 years in areas of lowest antimicrobial use and unchanged prevalence in areas of highest antimicrobial use. ${ }^{26}$

Restricting over-the-counter dispensation of antimicrobials without a prescription will eliminate a large source of antibiotic usage; studies indicate that more than $50 \%$ of antibiotics dispensed in developing countries are done without a prescription. ${ }^{12,13,27}$ Implementation would require governmental intervention to develop laws and regulations and to provide the means to enforce and monitor them. Although such a measure may reduce resistance, it is unclear if it may increase mortality from infectious disease by reducing the availability of antibiotics to poor and rural populations.

\section{Regulate the manufacture of antimicrobials in all countries to ensure purity and potency of antimicrobial pills}

As discussed, counterfeit and low-quality drugs affect not only the country of origin, but also any country with internet access. This makes it imperative that industrialized nations assist developing countries in regulating the manufacture of drugs. Developing countries should implement measures similar to those in the United States that ensure purity and potency of medications. Pharmaceutical companies can assist in this effort by providing the means to test for counterfeit drugs in each country.

The economic effects of this measure are unknown. Regulation poses a financial burden on the government that must enforce it and on the manufacturer who must prove compliance to standards. This, in turn, may lead to increased medication costs in developing countries and even further decrease access and compliance in poor populations.

\section{Provide developing countries access to more potent, albeit more expensive, antimicrobials to combat resistant organisms within the country}

As discussed, multidrug-resistant organisms are emerging at alarming rates in developing countries, yet these countries lack the financial resources to purchase newer, more potent antimicrobials to combat resistant strains. Instead they must rely on available, but less effective, antibiotics. ${ }^{2}$ This may lead to new, more resistant bacterial strains.

Wealthier nations, in cooperation with pharmaceutical companies, need to formulate a plan to make more potent antimicrobials available in developing countries. In turn, developing countries need to develop protocols to ensure that these antimicrobials are used appropriately to prevent resistance to these drugs.

\section{Develop protocols for high-dose, short-course antibiotic therapies to improve compliance and reduce resistance because of noncompliance}

A meta-analysis by Casey et $\mathrm{al}^{28}$ indicates that short-course cephalosporin treatment of group A streptococcal tonsillopharyngitis resulted in a superior bacterial cure rate when compared with 10 days of penicillin. In the Dominican Republic, not only did a high-dose, short-course amoxicillin ther- 
apy result in lower rates of resistant pneumococcal carriage in a pediatric population, but it also resulted in improved compliance. ${ }^{29}$ Shorter courses may not only improve compliance but may also help reduce the phenomenon of "leftover" medications and medication sharing that may be contributing to resistance.

Of course, development of such protocols needs to take into account toxicities and side effects that may result from higher doses of particular antibiotics. Furthermore, short-course, once-daily dosing regimens should be considered by drug manufacturers when developing new antimicrobials.

\section{Develop and promote community-wide education about the responsible use of antibiotics}

In 2002, Perz et $\mathrm{al}^{30}$ published a study demonstrating an $11 \%$ decrease in antibiotic prescription rates after a comprehensive, community-wide intervention involving education of providers, patients, and parents. A number of other studies and interventions also indicate that combined patient and provider education may result in reduced antibiotic use. ${ }^{31-33}$ However, when only the parent/patient was educated, antibiotic use remained unchanged. ${ }^{34,35}$ Therefore, education must occur community-wide for both patients and providers. Mass media educational campaigns may also have a role in community-wide education: one study suggests that a mass media intervention reduced antibiotic usage by $5.8 \% .^{36}$

\section{Areas for Further Study Within the United States}

The United States is lagging behind the international community in recognizing the role of poverty in antimicrobial resistance. To rectify the situation, studies analyzing socioeconomic and behavioral factors regarding antibiotic use need to be undertaken to recognize the impact of these factors on antimicrobial resistance and to identify areas for education and intervention.

Suggestions for further investigation include:

1. Determine antimicrobial practices in the lowincome population. Determine the duration of compliance to therapy, reasons for noncompliance, sources of medications, prevalence of medication sharing, and prevalence of use of drugs not made in the United States. Specific studies of low-income seniors and various lowincome ethnic groups should be undertaken because practices and beliefs may differ among these various populations.

2. Correlate resistance rates in communities that engage in self-medication (ie, medication sharing, purchasing of medications without a prescription, taking leftover antibiotics) with national resistance rates for various common organisms to determine how these practices impact resistance.

3. Examine perceptions regarding the role and benefit of antibiotics, including assessing the prevalence of common misperceptions regarding antibiotics. For example, within this author's primary care practice, patients have expressed the opinions that antibiotics provide symptom relief, they are necessary at the appearance of green sputum, and they can prevent progression of symptoms (ie, "nip it in the bud" mentality).

4. Continue to assess the efficacy of interventions involving community-wide education regarding appropriate antibiotic use and the problem of antimicrobial resistance.

Poverty has long been recognized in the international community as a driving force in antimicrobial resistance. But the role of poverty in antimicrobial resistance remains unrecognized in the United States, as evidenced by the dearth of related research. A problem that is not fully understood cannot be effectively solved. As the problem of antimicrobial resistance continues to escalate, it becomes more imperative that the effect of poverty on antimicrobial resistance be further studied, so that effective interventions can be implemented.

\section{Suggestions for the Individual Practitioner}

If the ideas presented in this paper are borne out by research, the following suggestions may be beneficial.

1. Obtain the latest bacterial susceptibility data from local hospitals and tailor antimicrobial choices accordingly, with a preference for narrow-spectrum drugs.

2. Educate patients about the dangers of antimicrobial resistance.

3. Advise patients on the importance of medication compliance and the need to take medications as prescribed for the duration prescribed. 
4. Encourage patients to dispose of any unused medications.

5. Educate patients on the dangers of using medications purchased over the internet or from a foreign country.

6. Query patients about their perceptions of the role of antibiotics and dispel any misperceptions that they may have.

7. Query patients about issues that may interfere with compliance (eg, ability to swallow pills, lifestyle issues that may impair ability to adhere to daily multidose regimens) and adjust the prescription accordingly.

8. Query patients about their ability to pay for medications at the time of visit.

9. Participate in or initiate research on the areas suggested in this article.

10. Encourage local medical societies, governmental agencies, lawmakers, and the media to make antimicrobial resistance a public health priority.

Primary care providers are on the front lines in the battle against antimicrobial resistance. Through action or inaction, primary care providers can either worsen the problem or contribute to the solution. Primary care providers can help solve the problem of antimicrobial resistance globally, nationally, and locally by recognizing the role of poverty and participating in advocacy, research, and education.

\section{References}

1. World Health Organization. Overcoming antimicrobial resistance. World Health Organization report on infectious diseases. World Health Organization; 2000.

2. Hart CA, Kariuki S. Antimicrobial resistance in developing countries. BMJ. 1998;317:647-50.

3. Okeke IN, Lamikanra A, Edelman R. Socioeconomic and behavioral factors leading to acquired bacterial resistance to antibiotics in developing countries. Emerg Infect Dis. 1999;5:18-27.

4. World Health Organization. World health report. Changing history. World Health Organization; 2004.

5. Murray CJL, Lopez AD. Mortality by cause for eight regions of the world: global burden of disease study. Lancet. 1997;349:1269-76.

6. Hossain MA, Rahman M, Ahmed QS, Malek MA, Sack RB, Albert MJ. Increasing frequency of mecillinam-resistant shigella isolates in urban Dhaka and rural Matlab, Bangladesh: a 6-year observation. J Antimicrob Chemother. 1998;42:99-102.

7. Mirza SH, Beeching NJ, Hart CA. Multi-drug resis- tant typhoid: a global problem. J Med Microbiol. 1996;44:317-9.

8. Klugman KP. Management of antibiotic-resistant pneumococcal infections. J Antimicrob Chemother. 1994;34:191-4.

9. Ross JDC. Fluoroquinolone resistance in gonorrhoea: how, where and so what. Int J STD AIDS. 1998;9:318-22.

10. Munoz R, Coffey TJ, Daniels M, et al. Intercontinental spread of a multi-resistant clone of serotype23F Streptococcus pneumoniae. J Infect Dis. 1991; 164:302-6.

11. Mamun KZ. Prevalence and genetics of resistance to commonly used antimicrobial agents in faecal enterobacteriaceae from children in Bangladesh $[\mathrm{PhD}$ thesis]. University of Liverpool; 1991.

12. Hossain MM, Glass RI, Khan MR. Antibiotic use in a rural community in Bangladesh. Int J Epidemiol. 1982;11:402-5.

13. Lansang MA, Lucas-Aquino R, Tupasi TE, et al. Purchase of antibiotics without prescription in $\mathrm{Ma}$ nila, the Philippines. Inappropriate choices and doses. J Clin Epidemiol. 1990;43:61-7.

14. DeNavas-Walt C, Proctor BD, Lee CH. Income, poverty and health insurance coverage in the United States: 2005. Washington, DC: U.S. Census Bureau; 2006:60-231.

15. Wan H, Sengupta M, Velkoff V, DeBarros K. $65+$ in the United States: 2005. Washington, DC: US Census Bureau; 2005:23-209.

16. Macias EP, Morales LS. Crossing the border for health care. J Health Care Poor Underserved. 2001; 12:77-87.

17. Larson E, Lin SX, Gomez-Duarte C. Antibiotic use in hispanic households, New York City. Emerg Infect Dis. 2003;9:1096-102.

18. McKee MD, Mills L, Mainous AG III. Antibiotic use for the treatment of upper respiratory infections in a diverse community. J Fam Pract. 1999;48:993-6.

19. Richman PB, Garra G, Eskin B, Nashed AH, Cody R. Oral antibiotic use without consulting a physician: a survey of ED patients. Am J Emerg Med. 2001;19: $57-60$.

20. Statement of William K. Hubbard, Associate Commissioner for Policy and Planning, Joint Hearing of the Subcommittee on Health Care and Subcommittee on International Trade, Committee on Finance, United States Senate. 2004 April 27.

21. Doern GV, Richter SS, Miller A, Miller N, Rice C, Heilmann K, Beekmann S. Antimicrobial resistance among Streptococcus pneumoniae in the United States: have we begun to turn the corner on resistance to certain antimicrobial classes? Clin Infect Dis. 2005; 41:139-48. Epub 2005 Jun 7.

22. Fox KK, Knapp JS, Holmes KK, et al. Antimicrobial resistance in Neisseria gonorrboeae in the United States, 1988-1994: the emergence of decreased sus- 
ceptibility to the fluoroquinolones. J Infect Dis. 1997;175:1396-403.

23. Centers for Disease Control and Prevention. Outbreak of multidrug-resistant Salmonella newportUnited States, January-April 2002. MMWR Morb Mortal Wkly Rep. 2002;51:545-8.

24. Vasquez Y, Hand WL. Antibiotic susceptibility patterns of community-acquired urinary tract infection isolates from female patients on the US (Texas)Mexico border. J Appl Res. 2004; 4(2):321-6.

25. Seppala H, Klaukka T, Vuopio-Varkila J, et al. The effect of changes in the consumption of macrolide antibiotics on erythromycin resistance in group A streptococci in Finland. N Engl J Med. 1997;337: 441-6.

26. Aranson VA, Sigurdsson JA, Erlendsdottir H, Gudmundsson S, Kristinsson KG. The role of antimicrobial use in the epidemiology of resistant pneumococci: a 10-year follow up. Microb Drug Resist. 2006;12:169-76.

27. Van Duong D, Binns CW, Van Le T. Availability of antibiotics as over-the-counter drugs in pharmacies: a threat to public health in Vietnam. Trop Med Int Health. 1997;2:1133-9.

28. Casey JR, Pichichero ME. Meta-analysis of shortcourse antibiotic treatment for group a streptococcal tonsillopharyngitis. Pediatr Infect Dis J. 2005;24: 909-17.

29. Schrag SJ, Pena C, Fenandez J, et al. Effect of short- course, high-dose amoxicillin therapy on resistant pneumococcal carriage. JAMA. 2001;286:49-56.

30. Perz JF, Craig AS, Coffey CS, et al. Changes in antibiotic prescribing for children after a community-wide campaign. JAMA. 2002;287:3103-9.

31. Finkelstein JA, Davis RL, Dowell SF, et al. Reducing antibiotic use in children: a randomized trial in 12 practices. Pediatrics. 2001;108:1-7.

32. Gonzales R, Steiner JF, Lum A, Barrett PH Jr. Decreasing antibiotic use in ambulatory practice: impact of a multidimensional intervention on the treatment of uncomplicated acute bronchitis in adults. JAMA. 1999;281:1512-9.

33. Trepka MJ, Belongia EA, Chyou PH, Davis JP, Schwartz B. The effect of a community intervention trial on parental knowledge and awareness of antibiotic resistance and appropriate antibiotic use in children. Pediatrics. 2001;107:E6.

34. Wheeler JG, Fair M, Simpson PM, Rowlands LA, Aitken ME, Jacobs RF. Impact of a waiting room videotape message on parent attitudes toward pediatric antibiotic use. Pediatrics. 2001;108:591-6.

35. Taylor JA, Kwan-Gett TS, McMahon EM Jr. Effectiveness of a parental educational intervention in reducing antibiotic use in children: a randomized controlled trial. Pediatr Infect Dis J. 2005;24:489_ 93.

36. Lambert MF, Masters GA, Brent SL. Can mass media campaigns change antimicrobial prescribing? A regional evaluation study. J Antimicrob Chemother. 2007;59:537-43. Epub 2007 Feb 5. 